\title{
Supplementary to "A note on asymptotic distributions in directed exponential random graph models with bi-degree sequences"
}

\author{
Jing Luo* Hong Qin ${ }^{\dagger} \quad$ Ting Yan ${ }^{\ddagger}, \sharp \quad$ Laala Zeyneb ${ }^{\S}$

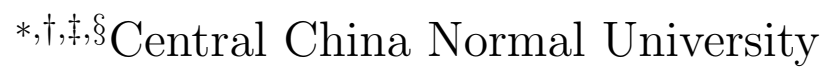

This is a supplementary material that contains proofs of the theorems 2 and 3 .

\section{$1 \quad$ Proof of Theorem 2}

Note that both $d_{i}=\sum_{j \neq i} a_{i, j}$ and $b_{i}=\sum_{i \neq j} a_{i, j}$ are sum of $n-1$ independent geometric random variables. Also note that $q_{n} \leq \bar{\alpha}_{i}^{*}+\bar{\beta}_{i}^{*} \leq Q_{n}$ and $V=F^{\prime}\left(\overline{\boldsymbol{\theta}}^{*}\right) \in \mathcal{L}_{n}(m, M)$, thus we have $(n-1) Q_{n}^{-2} \leq v_{i, i} \leq(n-1) q_{n}^{-2}$. Therefore, $v_{i, i}^{1 / 2}\left(d_{i}-\mathbb{E}\left(d_{i}\right)\right)$ is asymptotically standard normal if $Q_{n} / q_{n}=o\left(n^{1 / 2}\right)$. Similarly, $v_{n+j, n+j}^{-1 / 2}\left(b_{j}-\mathbb{E}\left(b_{j}\right)\right)$ is also asymptotically standard normal under the same condition. Similar to Proposition 2 in subsection 5.2 of the main texts, we have:

Proposition 1. Assume that $A \sim \mathbb{P}_{\boldsymbol{\theta}^{*}}$ and $Q_{n} / q_{n}=o\left(n^{1 / 2}\right)$, as $n \rightarrow \infty$, then $\bar{c}^{\top} S(\boldsymbol{g}-$ $\mathbb{E}(\boldsymbol{g}))$ is asymptotically normally distributed with mean zero and variance

$$
\sum_{i=1}^{\infty} \lambda_{i}^{2}+\sum_{i, j=1}^{\infty} \lambda_{i} \lambda_{j} H_{i} H_{j}+\sum_{i=1}^{\infty} \kappa_{i}^{2}+\sum_{i, j=1}^{\infty} \kappa_{i} \kappa_{j} H_{n+i} H_{n+j}-2 \sum_{i, j=1}^{\infty} \lambda_{i} \kappa_{j} H_{i} H_{n+j}
$$

where $\bar{\lambda}=\left(\lambda_{1} v_{11}^{1 / 2}, \ldots, \lambda_{n} v_{n n}^{1 / 2}\right)^{\top}, \bar{\kappa}=\left(\kappa_{1} v_{n+1, n+1}^{1 / 2}, \ldots, \kappa_{n-1} v_{2 n-1,2 n-1}^{1 / 2}\right)^{\top}$ and $\bar{c}=(\bar{\lambda}, \bar{\kappa})$.

Before proving Theorem 2, we present two lemmas. The proof of lemma 1 is similar to that of lemma 1 in subsection 5.2 of the main texts and we omit it.

${ }^{*}$ Department of Statistics ${ }^{*} \dagger, \ddagger, \S$ and Hubei Key Laboratory of Mathematical Sciences ${ }^{\sharp}$, Central China Normal University, Wuhan, 430079, China. Emails: *luojing0908@mails.ccnu.edu.cn, ${ }^{\dagger}$ qinhong@mail.ccnu.edu.cn, ${ }^{\ddagger}$ tingyanty@mail.ccnu.edu.cn, ${ }^{\S}$ zinebla91@hotmail.com. 
Lemma 1. Let $\bar{\lambda}=\left(\lambda_{1} v_{11}^{1 / 2}, \ldots, \lambda_{n} v_{n n}^{1 / 2}\right)^{\top}, \bar{\kappa}=\left(\kappa_{1} v_{n+1, n+1}^{1 / 2}, \ldots, \kappa_{n-1} v_{2 n-1,2 n-1}^{1 / 2}\right)^{\top}$ and $\bar{c}=$ $(\bar{\lambda}, \bar{\kappa})$. Then

$\operatorname{Var}\left[\bar{c}^{\top} W\{\boldsymbol{g}-\mathbb{E}(\boldsymbol{g})\}\right] \leq \frac{c_{1} M^{3}+3 M^{2} m^{2}}{m^{3}(n-1)} \times\left\{\left(\sum_{i=1}^{n}\left|\lambda_{i}\right|\right)^{2}+\left(\sum_{i=1}^{n-1}\left|\kappa_{i}\right|\right)^{2}+2\left(\sum_{i=1}^{n}\left|\lambda_{i}\right|\right)\left(\sum_{j=1}^{n-1}\left|\kappa_{j}\right|\right)\right\}$

where $m=1 / Q_{n}^{2}, M=1 / q_{n}^{2}$ and $c_{1}$ given in proposition 1 in subsection 5.1 of the main texts.

The following lemma is due to Theorem 3 in Yan et al. (2016).

Lemma 2. Assume that $\overline{\boldsymbol{\theta}}^{*}$ satisfies $q_{n} \leq \bar{\alpha}_{i}^{*}+\bar{\beta}_{j}^{*} \leq Q_{n}$ and $A \sim \mathbb{P}_{\overline{\boldsymbol{\theta}}^{*}}$, where $\mathbb{P}_{\overline{\boldsymbol{\theta}}^{*}}$ denote the probability distribution (1) of the main texts on $A$ under the parameter $\overline{\boldsymbol{\theta}}^{*}$. If $Q_{n} / q_{n}=o\left\{(n / \log n)^{1 / 18}\right\}$, then as $n$ goes to infinity, with probability approaching one, the $M L E \widehat{\boldsymbol{\theta}}$ exist and satisfies

$$
\left\|\widehat{\boldsymbol{\theta}}-\overline{\boldsymbol{\theta}}^{*}\right\|_{\infty}=O_{p}\left(\frac{Q_{n}^{9}(\log n)^{1 / 2}}{n^{1 / 2} q_{n}^{9}}\right)=o_{p}(1) .
$$

Further, if the MLE exists, it is unique.

Proof of Theorem 2. By Lemma 2, with probability approaching one, we have

$$
\hat{\rho}_{n}:=\max _{1 \leq i \leq 2 n-1}\left|\hat{\theta}_{i}-\bar{\theta}_{i}^{*}\right|=O\left(\frac{Q_{n}^{9}(\log n)^{1 / 2}}{n^{1 / 2} q_{n}^{9}}\right)
$$

The following calculations are based on the event (2). Let $\hat{\gamma}_{i, j}=\hat{\alpha}_{i}+\hat{\beta}_{j}-\bar{\alpha}_{i}^{*}-\bar{\beta}_{j}{ }^{*}$. For any $i \neq j$, a direct calculation gives

$$
\begin{aligned}
\frac{1}{\hat{\alpha}_{i}+\hat{\beta}_{j}}-\frac{1}{\bar{\alpha}_{i}^{*}+\bar{\beta}_{j}^{*}} & =\frac{\bar{\alpha}_{i}^{*}-\hat{\alpha}_{i}+\bar{\beta}_{j}^{*}-\hat{\beta}_{j}}{\left(\hat{\alpha}_{i}+\hat{\beta}_{j}\right)+\left(\bar{\alpha}_{i}^{*}+\bar{\beta}_{j}^{*}\right)} \\
& =\frac{\bar{\alpha}_{i}^{*}-\hat{\alpha}_{i}+\bar{\beta}_{j}^{*}-\hat{\beta}_{j}}{\left(\bar{\alpha}_{i}^{*}+\bar{\beta}_{j}^{*}\right)^{2}+\bar{\beta}_{j}^{*}} \frac{\bar{\alpha}_{i}+\hat{\beta}_{j}}{\hat{\alpha}_{i}^{*}-\hat{\alpha}_{i}+\bar{\beta}_{j}^{*}-\hat{\beta}_{j}} \\
& \left.=\bar{\alpha}_{i}^{*}+\bar{\beta}_{j}^{*}\right)^{2} \\
& h_{i, j}+\frac{\bar{\alpha}_{i}^{*}-\hat{\alpha}_{i}+\bar{\beta}_{j}^{*}-\hat{\beta}_{j}}{\left(\bar{\alpha}_{i}^{*}+\bar{\beta}_{j}^{*}\right)^{2}},
\end{aligned}
$$

where

$$
h_{i, j}=\frac{\hat{\gamma}_{i, j}^{2}}{\left(\hat{\alpha}_{i}+\hat{\beta}_{j}\right)\left(\bar{\alpha}_{i}^{*}+\bar{\beta}_{j}^{*}\right)^{2}},
$$

and $0 \leq \phi_{i, j} \leq 1$. By the likelihood equations (4) in subsection 2.2 of the main texts, we 
have

$$
\mathbf{g}-\mathbb{E}(\mathbf{g})=V\left(\widehat{\boldsymbol{\theta}}-\overline{\boldsymbol{\theta}}^{*}\right)+\mathbf{h},
$$

where $\mathbf{h}=\left(h_{1}, \ldots, h_{2 n-1}\right)^{\top}$ and

$$
\begin{aligned}
h_{i} & =\sum_{k=1, k \neq i}^{n} h_{i, k}, i=1, \ldots, n, \\
h_{n+i} & =\sum_{k=1, k \neq i}^{n} h_{k, i}, i=1, \ldots, n-1 .
\end{aligned}
$$

Equivalently,

$$
\widehat{\boldsymbol{\theta}}-\overline{\boldsymbol{\theta}}^{*}=V^{-1}(\mathbf{g}-\mathbb{E}(\mathbf{g}))+V^{-1} \mathbf{h}
$$

By the definition of $\hat{\rho}_{n}$, we have

$$
\left|h_{i, j}\right| \leq \frac{4 \hat{\rho}_{n}^{2}}{q_{n}^{2}\left(q_{n}-\hat{\rho}_{n}\right)}, \quad\left|h_{i}\right| \leq \sum_{j \neq i}\left|h_{i, j}\right| \leq \frac{4(n-1) \hat{\rho}_{n}^{2}}{q_{n}^{2}\left(q_{n}-\hat{\rho}_{n}\right)} .
$$

Note that $(S \mathbf{h})_{i}=h_{i} / v_{i, i}+(-1)^{1_{\{i>n\}}} h_{2 n} / v_{2 n, 2 n},(n-1) Q_{n}^{-2} \leq v_{i, i} \leq(n-1) q_{n}^{-2}$ and $\left(V^{-1} \mathbf{h}\right)_{i}=(S \mathbf{h})_{i}+(W \mathbf{h})_{i}$. By direct calculations, we have

$$
\begin{aligned}
\left|\left(V^{-1} \mathbf{h}\right)_{i}\right|=\left|(S \mathbf{h})_{i}\right|+\left|(W \mathbf{h})_{i}\right| & \leq \frac{\left|h_{i}\right|}{v_{i, i}}+\frac{\left|h_{2 n}\right|}{v_{2 n, 2 n}}+\left\{\|W\|_{\infty} \times\left[(2 n-1) \max _{i}\left|h_{i}\right|\right]\right\} \\
& \leq \frac{8 Q_{n}^{2}{\hat{\rho_{n}}}^{2}}{q_{n}^{3}}+\frac{8 c_{1} Q_{n}^{6}{\hat{\rho_{n}}}^{2}}{q_{n}^{7}} \\
& =O\left(\frac{\log (n) Q_{n}^{24}}{n q_{n}^{21}}\right)
\end{aligned}
$$

where $c_{1}$ given in proposition 1 in subsection 5.1 of the main texts.

Note that $\bar{c}=(\bar{\lambda}, \bar{\kappa}), \bar{\lambda}=\left(\lambda_{1} v_{11}^{1 / 2}, \ldots, \lambda_{n} v_{n n}^{1 / 2}\right)^{\top}$ and $\bar{\kappa}=\left(\kappa_{1} v_{n+1, n+1}^{1 / 2}, \ldots, \kappa_{n-1} v_{2 n-1,2 n-1}^{1 / 2}\right)^{\top}$. Consequently, if $\sum_{i=1}^{\infty}\left|\lambda_{i}\right|<\infty, \sum_{i=1}^{\infty}\left|\kappa_{i}\right|<\infty$ and $Q_{n} / q_{n}=o\left(n^{1 / 50} /(\log n)^{1 / 25}\right)$, then as $n \rightarrow \infty$,

$$
\begin{array}{r}
\left|\sum_{i=1}^{2 n-1} \bar{c}_{i}\left(V^{-1} \mathbf{h}\right)_{i}\right|=\left|\sum_{i=1}^{n} \lambda_{i} \sqrt{v_{i, i}}\left(V^{-1} \mathbf{h}\right)_{i}+\sum_{i=1}^{n-1} \kappa_{i} \sqrt{v_{n+i, n+i}}\left(V^{-1} \mathbf{h}\right)_{n+i}\right| \\
=O\left(\frac{\log (n) Q_{n}^{24}}{n^{1 / 2} q_{n}^{23}}\left(\sum_{i=1}^{n}\left|\lambda_{i}\right|+\sum_{i=1}^{n-1}\left|\kappa_{i}\right|\right)\right) \rightarrow 0
\end{array}
$$

In view of (4) and Lemma 1 , if $Q_{n} / q_{n}=o\left(n^{1 / 50} /(\log n)^{1 / 25}\right)$ and condition (2) of the main 
texts hold, then

$$
\begin{aligned}
\bar{c}^{\top}\left(\widehat{\boldsymbol{\theta}}-\overline{\boldsymbol{\theta}}^{*}\right) & =\bar{c}^{\top} S(g-\mathbb{E}(\mathbf{g}))+\bar{c}^{\top} W\{\mathbf{g}-\mathbb{E}(\mathbf{g})\}+\left|\sum_{i} \bar{c}_{i}\left(V^{-1} \mathbf{h}\right)_{i}\right| \\
& =\bar{c}^{\top} S(\mathbf{g}-\mathbb{E}(\mathbf{g}))+o_{p}(1) .
\end{aligned}
$$

Theorem 2 is immediately comes form Proposition 1.

\section{Proof of theorem 3}

Note that both $d_{i}=\sum_{j \neq i} a_{i, j}$ and $b_{i}=\sum_{i \neq j} a_{i, j}$ are sums of $n-1$ independent geometric random variables. Also note that $q_{n} \leq \bar{\alpha}_{i}^{*}+\bar{\beta}_{i}^{*} \leq Q_{n}$ and $V=F^{\prime}\left(\overline{\boldsymbol{\theta}}^{*}\right) \in \mathcal{L}_{n}(m, M)$, here $m=\frac{e^{Q_{n}}}{\left(e^{Q_{n}}-1\right)^{2}}$ and $M=\frac{e^{q_{n}}}{\left(e^{q_{n}}-1\right)^{2}}$, thus we have

$$
\begin{aligned}
& \frac{e^{Q_{n}}}{\left(e^{Q_{n}}-1\right)^{2}} \leq v_{i, j} \leq \frac{e^{q_{n}}}{\left(e^{q_{n}}-1\right)^{2}}, i=1, \ldots, n, j=n+1, \ldots, 2 n, j \neq n+i, \\
& \frac{(n-1) e^{Q_{n}}}{\left(e^{Q_{n}}-1\right)^{2}} \leq v_{i, i} \leq \frac{(n-1) e^{q_{n}}}{\left(e^{q_{n}}-1\right)^{2}}, i=1, \ldots, 2 n .
\end{aligned}
$$

Note that if $e^{Q_{n} / 2} / q_{n}=o\left(n^{1 / 2}\right), v_{i, i}^{1 / 2}\left(d_{i}-\mathbb{E}\left(d_{i}\right)\right)(i=1, \ldots, n)$ is asymptotically standard normal and $v_{n+j, n+j}^{-1 / 2}\left(b_{j}-\mathbb{E}\left(b_{j}\right)\right)(j=1, \ldots, n)$ is asymptotically standard normal. Similar to Proposition 2 in subsection 5.2 of the main texts, we have:

Proposition 2. Assume that $e^{Q_{n} / 2} / q_{n}=o\left(n^{1 / 2}\right)$, as $n \rightarrow \infty$, then $\bar{c}^{\top} S(\boldsymbol{g}-\mathbb{E}(\boldsymbol{g}))$ is asymptotically normally distributed with mean zero and variance

$\sum_{i=1}^{\infty} \lambda_{i}^{2}+\sum_{i, j=1}^{\infty} \lambda_{i} \lambda_{j} H_{i} H_{j}+\sum_{i=1}^{\infty} \kappa_{i}^{2}+\sum_{i, j=1}^{\infty} \kappa_{i} \kappa_{j} H_{n+i} H_{n+j}-2 \sum_{i, j=1}^{\infty} \lambda_{i} \kappa_{j} H_{i} H_{n+j}$, where $\bar{\lambda}=\left(\lambda_{1} v_{11}^{1 / 2}, \ldots, \lambda_{n} v_{n n}^{1 / 2}\right)^{\top}, \bar{\kappa}=\left(\kappa_{1} v_{n+1, n+1}^{1 / 2}, \ldots, \kappa_{n-1} v_{2 n-1,2 n-1}^{1 / 2}\right)^{\top}$ and $\bar{c}=(\bar{\lambda}, \bar{\kappa})$.

Before proving Theorem 3, we present two lemmas. The proof lemma 3 is similar to that of lemma 1 in subsection 5.2 of the main texts and we omit it. Lemma 4 is due to Theorem 5 in Yan et al. (2016).

Lemma 3. Let $\bar{\lambda}=\left(\lambda_{1} v_{11}^{1 / 2}, \ldots, \lambda_{n} v_{n n}^{1 / 2}\right)^{\top}, \bar{\kappa}=\left(\kappa_{1} v_{n+1, n+1}^{1 / 2}, \ldots, \kappa_{n-1} v_{2 n-1,2 n-1}^{1 / 2}\right)^{\top}$ and $\bar{c}=$ $(\bar{\lambda}, \bar{\kappa})$. Then

$\operatorname{Var}\left[\bar{c}^{\top} W\{\boldsymbol{g}-\mathbb{E}(\boldsymbol{g})\}\right] \leq \frac{c_{1} M^{3}+M^{2} m}{m^{3}(n-1)} \times\left\{\left(\sum_{i=1}^{n}\left|\lambda_{i}\right|\right)^{2}+\left(\sum_{i=1}^{n-1}\left|\kappa_{i}\right|\right)^{2}+2\left(\sum_{i=1}^{n}\left|\lambda_{i}\right|\right)\left(\sum_{j=1}^{n-1}\left|\kappa_{j}\right|\right)\right\}$

where $m=\frac{e^{Q_{n}}}{\left(e^{Q_{n}}-1\right)^{2}}, M=\frac{e^{q_{n}}}{\left(e^{q_{n}}-1\right)^{2}}$ and $c_{1}$ given in proposition 1 in subsection 5.1 of the main texts. 
Lemma 4. Assume that $\overline{\boldsymbol{\theta}}^{*}$ satisfies $q_{n} \leq \bar{\alpha}_{i}^{*}+\bar{\beta}_{j}^{*} \leq Q_{n}$ for all $i \neq j$ and $A \sim \mathbb{P}_{\overline{\boldsymbol{\theta}}^{*}}$, where $\mathbb{P}_{\overline{\boldsymbol{\theta}}^{*}}$ denote the probability distribution (1) of the main texts on $A$ under the parameter $\overline{\boldsymbol{\theta}}^{*}$. If $\left(1+q_{n}^{-11}\right) e^{6 Q_{n}}=o\left(n^{1 / 2} /(\log n)^{1 / 2}\right)$, then as $n$ goes to infinity, with probability approaching one, the MLE $\widehat{\boldsymbol{\theta}}$ exist and satisfies

$$
\left\|\widehat{\boldsymbol{\theta}}-\overline{\boldsymbol{\theta}}^{*}\right\| \infty=O_{p}\left(e^{3 Q_{n}}\left(1+\frac{1}{q_{n}^{5}}\right) \sqrt{\frac{\log n}{n}}\right)=o_{p}(1)
$$

Further, if the MLE exists, it is unique.

Proof of Theorem 3. By Lemma 4, with probability approaching one, we have

$$
\hat{\rho}_{n}:=\max _{1 \leq i \leq 2 n-1}\left|\hat{\theta}_{i}-\bar{\theta}_{i}^{*}\right|=O\left(e^{3 Q_{n}}\left(1+\frac{1}{q_{n}^{5}}\right) \sqrt{\frac{\log n}{n}}\right) .
$$

The following calculations are based on the event (6). Let $\hat{\gamma}_{i, j}=\hat{\alpha}_{i}+\hat{\beta}_{j}-\bar{\alpha}_{i}^{*}-\bar{\beta}_{j}{ }^{*}$. By Taylor's expansion, for any $i \neq j$, we have

$$
\frac{1}{e^{\hat{\alpha}_{i}+\hat{\beta}_{j}}-1}-\frac{1}{e^{\bar{\alpha}_{i}^{*}+\bar{\beta}_{j}^{*}}-1}=-\frac{e^{\bar{\alpha}_{i}^{*}+\bar{\beta}_{j}^{*}}}{\left(e^{\bar{\alpha}_{i}^{*}+\bar{\beta}_{j}^{*}}-1\right)^{2}} \hat{\gamma}_{i, j}+h_{i, j},
$$

where

$$
h_{i, j}=-\frac{e^{\bar{\alpha}_{i}^{*}+\bar{\beta}_{j}^{*}+\eta_{i, j} \hat{\gamma}_{i, j}}\left(1+e^{\bar{\alpha}_{i}^{*}+\bar{\beta}_{j}^{*}+\eta_{i, j} \hat{\gamma}_{i, j}}\right)}{\left(e^{\bar{\alpha}_{i}^{*}+\bar{\beta}_{j}^{*}+\eta_{i, j} \hat{\gamma}_{i, j}}-1\right)^{3}},
$$

and $0 \leq \eta_{i, j} \leq 1$. It is not difficult to verify that

$$
\mathbf{g}-\mathbb{E}(\mathbf{g})=V\left(\widehat{\boldsymbol{\theta}}-\overline{\boldsymbol{\theta}}^{*}\right)+\mathbf{h},
$$

where $\mathbf{h}=\left(h_{1}, \ldots, h_{2 n-1}\right)^{\top}$ and

$$
\begin{aligned}
h_{i} & =\sum_{k=1, k \neq i}^{n} h_{i, k}, i=1, \ldots, n, \\
h_{n+i} & =\sum_{k=1, k \neq i}^{n} h_{k, i}, i=1, \ldots, n-1 .
\end{aligned}
$$

Equivalently,

$$
\widehat{\boldsymbol{\theta}}-\overline{\boldsymbol{\theta}}^{*}=V^{-1}(\mathbf{g}-\mathbb{E}(\mathbf{g}))+V^{-1} \mathbf{h} .
$$

Since $\bar{\alpha}_{i}^{*}+\bar{\beta}_{j}^{*}>0, q_{n}-\hat{\rho}_{n}<\alpha_{i}+\beta_{j}+\eta_{i, j} \hat{\gamma}_{i, j} \leq Q_{n}+q_{n}$ and $\hat{\rho}_{n}$ is sufficiently small, by 
the definition of $\hat{\rho}_{n}$, we have

$$
\left|h_{i, j}\right| \leq \frac{e^{\left(q_{n}-\hat{\rho}_{n}\right)}\left(1+e^{\left(q_{n}-\hat{\rho}_{n}\right)}\right)}{\left(e^{\left(q_{n}-\hat{\rho}_{n}\right)}-1\right)^{3}} \hat{\rho}_{n}^{2}, \quad\left|h_{i}\right| \leq \sum_{j \neq i}\left|h_{i, j}\right| \leq(n-1) \frac{e^{\left(q_{n}-\hat{\rho}_{n}\right)}\left(1+e^{\left(q_{n}-\hat{\rho}_{n}\right)}\right)}{\left(e^{\left(q_{n}-\hat{\rho}_{n}\right)}-1\right)^{3}} \hat{\rho}_{n}^{2} .
$$

Note that $(S \mathbf{h})_{i}=h_{i} / v_{i, i}+(-1)^{1_{\{i>n\}}} h_{2 n} / v_{2 n, 2 n}$. By direct calculations, we have

$$
\begin{aligned}
\left|\left(V^{-1} \mathbf{h}\right)_{i}\right| & =\left|(S \mathbf{h})_{i}\right|+\left|(W \mathbf{h})_{i}\right| \leq \frac{\left|h_{i}\right|}{v_{i, i}}+\frac{\left|h_{2 n}\right|}{v_{2 n, 2 n}}+\left\{\|W\|_{\infty} \times\left[(2 n-1) \max _{i}\left|h_{i}\right|\right]\right\} \\
& \leq O\left(\frac{\log (n)}{n} \times e^{9 Q_{n}}\left(1+q_{n}^{-15}\right)\right)
\end{aligned}
$$

Let $\bar{c}=(\bar{\lambda}, \bar{\kappa}), \bar{\lambda}=\left(\lambda_{1} v_{11}^{1 / 2}, \ldots, \lambda_{n} v_{n n}^{1 / 2}\right)^{\top}$ and $\bar{\kappa}=\left(\kappa_{1} v_{n+1, n+1}^{1 / 2}, \ldots, \kappa_{n-1} v_{2 n-1,2 n-1}^{1 / 2}\right)^{\top}$. Consequently, if $\sum_{i=1}^{\infty}\left|\lambda_{i}\right|<\infty, \sum_{i=1}^{\infty}\left|\kappa_{i}\right|<\infty$ and $e^{9 Q_{n}}\left(1+q_{n}^{-15}\right)=o\left(n^{1 / 2} / \log n\right)$, then as $n \rightarrow \infty$,

$$
\begin{aligned}
\left|\sum_{i=1}^{2 n-1} \bar{c}_{i}\left(V^{-1} \mathbf{h}\right)_{i}\right| & =\left|\sum_{i=1}^{n} \lambda_{i} \sqrt{v_{i, i}}\left(V^{-1} \mathbf{h}\right)_{i}+\sum_{i=1}^{n-1} \kappa_{i} \sqrt{v_{n+i, n+i}}\left(V^{-1} \mathbf{h}\right)_{n+i}\right| \\
\leq & O\left(\frac{\log (n)}{n^{1 / 2}} \times e^{9 Q_{n}}\left(1+q_{n}^{-15}\right)\left(\sum_{i=1}^{n}\left|\lambda_{i}\right|+\sum_{i=1}^{n-1}\left|\kappa_{i}\right|\right)\right) \rightarrow 0
\end{aligned}
$$

In view of $(7)$ and Lemmas 3 and 4 , if $e^{9 Q_{n}}\left(1+q_{n}^{-15}\right)=o\left(n^{1 / 2} / \log n\right)$ and condition(2) of the main texts hold, then

$$
\begin{aligned}
\bar{c}^{\top}\left(\widehat{\boldsymbol{\theta}}-\overline{\boldsymbol{\theta}}^{*}\right) & =\bar{c}^{\top} S(\mathbf{g}-\mathbb{E}(\mathbf{g}))+\bar{c}^{\top} W\{\mathbf{g}-\mathbb{E}(\mathbf{g})\}+\left|\sum_{i} \bar{c}_{i}\left(V^{-1} \mathbf{h}\right)_{i}\right| \\
& =\bar{c}^{\top} S(\mathbf{g}-\mathbb{E}(\mathbf{g}))+o_{p}(1)
\end{aligned}
$$

Theorem 3 is immediately comes from Proposition 2.

\section{References}

Yan, T., Leng, C., and Zhu, J. (2016). Asymptotics in directed exponential random graph models with an increasing bi-degree sequence. The Annals of Statistics, 44(1):31-57. 